\title{
Astronomie et mathématiques anciennes et classiques
}

\author{
Roshdi Rashed*
}

\begin{abstract}
Résumé: On examine les disciplines mathématiques qui doivent leur existence à l'astronomie ancienne et classique. Les exemples de la géométrie des coniques et des recherches sur le gnomon, des méthodes d'approximation numérique et de l'établissement de tables astronomiques, de la trigonométrie et l'étude des mouvements célestes, sont rapidement évoqués. On s'attache plus particulièrement à deux autres chapitres des mathématiques : les recherches isopétimétriques, tout d'abord, qui doivent leur origine aux conceptions cosmologiques sur le cercle et la sphère, et ont suscité les études sur l'extrémalité (Ibn al-Haytam), et donné lieu à la géométrie métrique. Les problèmes de projection de la sphère qui sont à l'origine de la géométrie des positions, d'autre part, qui doivent leur développement initial à la construction des astrolabes étendus ensuite au point de vue projectif d'une manière plus générale (en particulier, Ibn Sahl, al-Quhi). Ces domaines des mathématiques se sont développés ultérieurement en s'affranchissant de ces conditions d'origine.

Abstract: We examine the mathematical disciplines that owe their existence to ancient and classical astronomy. The exemples of the geometry of conics with the researches on the gnomon, of the methods of numerical approximations with the setting of astronomical tables, of trigonometry with the study of celestial motions, are briefly evoked. We emphasize two other chapters of mathematics. Firstly, the studies on isoperimetrics, originated in cosmological conceptions on the circle and the sphere, have generated the studies on extremality (Ibn al-Haytham) and given rise to metrical geometry. Secondly, the problems of projection of the sphere, which generated geometry of positions, have been initially impulsed by the construction of astrolabs; the projective problem has been considered afterwards in a more general way (in particular, Ibn Sahl, al-Quhi). These domains of mathematics have developed further independently of the conditions of their origins.
\end{abstract}

\section{L'HISTOIRE DES MATHÉMATIQUES est intimement liée à l'histoi-} re de la cosmologie et de l'astronomie. Cette affirmation va de soi lorsqu'il s'agit de l'histoire récente: le renouvellement des notions astronomiques a, chaque fois, des répercussions sur les mathématiques, et il n'est pas moins vrai que les nouvelles méthodes mathématiques ont permis l'élaboration des conceptions astronomiques. On sait combien ces rapports entre astronomie et mathématiques sont essentiels à l'histoire du calcul différentiel, de la géométrie différentielle, du calcul des probabilités, des statistiques, entre autres disciplines mathématiques. Par

\footnotetext{
* Directeur de recherche, Centre d'histoire des sciences et des philosophies arabes et médiévales, CNRS et Université Paris 7 - Denis Diderot - 7, rue Guy Môquet B.P. No. 8 - 94801 Villejuif.
} 
conséquent, l'historien des mathématiques récentes ne peut ni ne doit faire l'économie de l'examen de ces rapports.

En est-il de même pour l'historien des mathématiques anciennes et classiques? Autrement dit, ces liens étroits entre mathématiques et astronomie étaient-ils également, pour les mathématiciens de l'antiquité et du moyen-âge, l'horizon privilégié de leur recherche? S'il s'agit de l'antiquité, la question risque de s'étendre et de se compliquer davantage, puisqu'elle peut porter sur l'origine historique, mais aussi phénoménologique, de certaines branches des mathématiques. On songera alors à ce qu'aurait pu être la géométrie dans l'espace, par exemple, si l'astronomie positive n'avait jamais vu le jour. On se demandera aussi ce qu'il serait advenu d'une telle discipline si l'homme avait négligé d'observer le ciel pour décrire, et, éventuellement, mesurer, les apparences. Ces questions d'"origine" ne peuvent à l'évidence être résolues à l'aide de la seule description, fût-elle eidétique: leur solution appelle une restitution historique, qui n'est pas faite, tant s'en faut. La seule stratégie qui se dessine pour approcher ce but lointain est d'examiner les disciplines mathématiques qui doivent peu ou prou leur existence à l'astronomie ancienne et classique. Parmi ces disciplines, on rencontre aussi bien la géométrie des positions que la géométrie métrique, l'analyse numérique et la trigonométrie.

Venons-en d'abord, et très brièvement, à l'une des théories mathématiques parmi les plus importantes des mathématiques grecques: la théorie des coniques telle qu'elle se présente dans le livre d'Apollonius. L'origine de cette théorie semble se fondre dans deux traditions de recherche relatives à deux espèces d"“organon" au sens premier du terme, toutes deux en quelconque relation à l'astronomie. La première tradition, ainsi que j'ai pu l'établir, porte sur les miroirs ardents. Il s'agissait, dans le milieu de Conon d'Alexandrie, de Dosithée, de Dioclès, etc., de construire des miroirs ardents paraboliques. On exigeait par exemple qu'ils embrasent selon un cercle, ou qu'ils embrasent sans être dirigés vers le soleil, fixes et indiquant l'heure sans gnomon; problème optico-astronomique d'une difficulté redoutable. Dans la seconde tradition s'inscrivent les recherches menées sur le gnomon. Il s'agit d'un gnomon d'une longueur donnée, et de position fixe. Le plan sur lequel tombe l'ombre de l'extrémité de ce gnomon est perpendiculaire à celui-ci, et on cherche à déterminer la grandeur de l'ombre en fonction du temps. On peut alors montrer que l'on retrouvera dans ces conditions les sections coniques, selon la conception de Menechme. C'est ce qu'a fait $O$. Neugebauer, lorsqu'il a proposé, en des termes certes spéculatifs, mais non point invraisemblables, de fixer là l'origine des coniques. Il apparaît donc que les instruments et les problèmes astronomiques sont l'une 
des origines de la théorie des coniques, laquelle semble prendre son autonomie vers le $\mathrm{III}^{\mathrm{e}}$ siècle avant notre ère.

Mais le rôle de l'astronomie n'est pas moins important si l'on considère la formation et le développement de l'analyse numérique. Les astronomes ont très tôt cherché des méthodes pour établir et utiliser des tables astronomiques et trigonométriques, et ils ont à cette occasion développé des concepts et des méthodes d'interpolation. L'interpolation linéaire, aussi ancienne que l'astronomie babylonienne, était le point de départ de toutes les tentatives ultérieures: la voie naturelle consista en effet à améliorer cette interpolation. C'est en tous cas celle qu'a suivie Brahmagupta pour l'élaboration d'une méthode d'interpolation parabolique. C'est la même voie qu'a empruntée un anonyme indien pour donner une autre méthode d'interpolation quadratique, dite Sankalt. Les astronomes vont, à partir du $\mathrm{IX}^{\mathrm{e}}$ siècle, multiplier le nombre des méthodes d'interpolation quadratique, les comparer pour choisir celle qui approche le mieux la fonction considérée. Cette comparaison a donné sa première dimension théorique à une recherche essentiellement "expérimentale", pour ainsi dire. C'est avec ces astronomes-mathématiciens, comme al-Biruni, que l'on assiste à l'émergence du calcul aux différences finies ${ }^{1}$

Il en est de même, comme chacun sait, pour la trigonométrie. À l'origine, elle n'a pas de nom, elle se présente comme un auxiliaire de l'étude des mouvements célestes. On attribue à Hipparque la première table des cordes; mais ce sont les astronomes de l'Inde qui, vers le $\mathrm{VI}^{\mathrm{e}}$ siècle de notre ère, ont remplacé la corde de l'arc double par sa moitié. Il fallut cependant attendre le $\mathrm{x}^{\mathrm{e}}$ siècle avant que les astronomes obtiennent les premières relations du triangle sphérique: c'est vers cette époque que la trigonométrie émerge comme science autonome, sous forme de compositions propres et indépendantes. On rencontre entre autres contributions la découverte de méthodes pour le calcul de $\sin 1^{\circ} 2$

À peine rappelés, ces exemples suffisent à désigner l'astronomie ancienne comme étant à l'origine, partiellement tout au moins, des disciplines mathématiques, lesquelles toutefois ne se constituent comme telles qu'une fois rompues les attaches qui les unissaient à l'astronomie. Pour illustrer cette idée, j'examinerai, sans m'étendre davantage, deux autres chapitres des mathématiques ạciennes et classiques: les recherches isopérimétriques et les études projectives.

1 R. Rashed, "Al-Samaw'al, al-Biruni et Brahmagupta: sur les méthodes d'interpolation", Arabic Sciences and Pbilosopby: a Historical Journal, 1 (1991), p. 100-50.

2 M.-Th. Debarnot, "Trigonométrie", dans R. Rashed (éd.), Histoire des sciences arabes, Paris, 1997, Vol. II, p. 163-98. 
Venons-en d'abord au problème isopérimétrique qui a surgi tout naturellement pour ainsi dire dans le cadre de la cosmologie babylonienne et grecque.

Montrer que, parmi les domaines du plan ayant un périmètre donné, le disque a la plus grande aire; et que, de tous les solides de l'espace ayant la même aire latérale, la sphère a le plus grand volume: d'après les témoignages tardifs, ce résultat semble être un acquis ancien. C'est en tout cas l'opinion de Simplicius. Mais de l'avis de tous, c'est à Zénodore que revient d'avoir traité le problème et d'avoir établi la démonstration, dans son traité perdu Sur les figures isopérimétriques. Le problème n'a d'ailleurs pas cessé d'intéresser les astronomes et les mathématiciens: Héron d'Alexandrie, Ptolémée, Pappus, Théon d'Alexandrie. En fait, c'est Ptolémée et son Almageste qui ont été la source de ce problème pendant plus d'un millénaire.

Dans l'Almageste en effet, à l'appui de sa thèse de la sphéricité, si importante pour sa cosmologie et son astronomie, Ptolémée rappelle le précédent résultat comme un acquis, et écrit:

"Puisque, parmi les figures différentes mais isopérimétriques, celles qui ont le plus de côtés sont plus grandes, parmi les figures planes, c'est le cercle qui est la plus grande, et parmi les solides, c'est la sphère qui a le plus grand volume et le ciel est le plus grand corps." 3

À partir de Théon d'Alexandrie et de Pappus, les commentateurs de l' $A l$ mageste ne pouvaient désormais faire silence sur cette affirmation, et se devaient d'en apporter la preuve. Tout un courant de recherche s'enclenche donc, afin d'établir cette conception cosmologique. Citons simplement Théon d'Alexandrie:

"Nous allons le prouver d'une manière abrégée, tirée de la démonstration de Zénodore dans son traité des figures isopérimètres." 4

3 J.L. Heiberg, Claudii Ptolemaei opera quae extant omnia. I. Syntaxis mathematica (Leipzig, 1898), p. 13, 1. 16-9.

4 A. Rome, Commentaires de Pappus et de Théon d'Alexandrie sur l'Almageste, texte établi et annoté, T. II: Théon d'Alexandrie, Commentaire sur les livres 1 et 2 de l'Almageste (Vatican, 1936), p. 33. 
Dans les premières décades du $\mathrm{IX}^{\mathrm{e}}$ siècle, l'Almageste aussi bien que le Commentaire de Théon d'Alexandrie étaient rendus en arabe. Tous les commentateurs arabes de l'Almageste s'intéressent à ce problème, et deux courants de recherche mathématique s'engagent alors pour établir l'affirmation de Ptolémée. Le premier courant est représenté par un mathématicien-astronome du milieu du $\mathrm{x}^{\mathrm{e}}$ siècle: al-Khazin. Son idée directrice est de placer ce problème dans un contexte plus général, celui de toutes les figures convexes, et de montrer que, parmi les figures convexes d'un type donné (triangle, losange, parallélogramme, etc.), celle dont la symétrie est la plus parfaite réalise un extremum pour un certain paramètre (aire, rapport d'aire, périmètres, etc.) ${ }^{5}$. On procède alors de la manière suivante: on fixe un paramètre et on fait varier la figure en la symétrisant par rapport à une certaine droite. Par exemple, on fixe le périmètre d'un parallélogramme, et on transforme ce parallélogramme en un losange en symétrisant par rapport à une diagonale: l'aire augmente au cours du processus. La démarche concrète d'al-Khazin s'ordonne de la manière suivante: 1) il commence par comparer les polygones réguliers de même périmètre et montre que celui qui a le plus grand nombre de côtés a la plus grande aire. 2) il compare ensuite un polygone régulier circonscrit à un cercle et un cercle de même périmètre. Démarche "statique", en ce sens que l'on a d'une part le polygone donné, et, de l'autre, le cercle. Sans vouloir entrer dans les détails, disons simplement qu'alKhazin termine son traité une fois établies la propriété isopérimétrique et la propriété isépiphanique, c'est-à-dire ce qui est requis par la cosmologie.

La seconde tendance est représentée par un autre mathématicien, mort après 1040 , le célèbre Ibn al-Haytham ${ }^{6}$. Il entendait à l'évidence donner une démonstration "dynamique" de ces deux propriétés. C'est alors qu'il a rédigé un traité qui fut à l'avant-garde de la recherche mathématique de l'époque, mais qui conserva ce poste durant bien des siècles. Ibn al-Haytham commence par régler rapidement le cas des figures planes. Tout comme son prédécesseur alKhazin, il compare des polygones réguliers de même périmètre, et d'un nombre de côtés différents, et démontre

1) Soient deux polygones réguliers de même périmètre, celui qui a le plus grand nombre de côtés a la plus grande aire.

2) Si un cercle et un polygone régulier ont le même périmètre, alors l'aire du cercle est plus grande que celle du polygone.

$5 \mathrm{R}$. Rashed, Les Matbématiques infinitésimales du IX $X^{e}$ au XI siècle, Vol. I: Fondateurs et commentateurs: Banu Musa, Thabit ibn Qurra, Ibn Sinan, al-Khazin, al-Qubi, Ibn al-Samb, Ibn Hud, Londres, 1996.

6 R. Rashed, Les Matbématiques infinitésimales du IX'e au XIe siècle, Vol. II: Ibn al-Haytbam, Londres, 1993. 

"étant donné deux points $A$ et $B$ dans un plan vertical, trouver le chemin $A M B$ qu'un point mobile $M$ parcourt de $A$ à $B$ par vertu de son poids dans le plus court temps possible."7

Nous sommes déjà là sur un autre terrain, celui du calcul des variations naissant, différent de celui qu'avait investi la recherche issue du problème cosmologique. Cette dernière recherche s'était déjà épuisée, non pas après Ibn alHaytham, mais, si j'ose dire, dès le milieu de son livre.

Le second exemple sur lequel je voudrais rapidement m'arrêter ne relève plus de la géométrie métrique, mais de la géométrie des positions. Cette fois encore, c'est de Ptolémée qu'il faut partir, de son Planisphère conservé dans une version arabe, ainsi que dans une traduction latine de cette dernière. Voici le début du livre de Ptolémée:

"Puisqu'il est possible, oh Syros, et utile dans plusieurs chapitres, de tracer sur une surface plane les cercles qui se trouvent sur la sphère solide comme s'ils étaient plans, j'ai pensé qu'il est de mon devoir vis-à-vis de la science d'écrire un livre pour celui qui veut connaître cela, dans lequel je montre brièvement de quelle manière il est possible de tracer le cercle de l'orbe incliné (l'écliptique), les cercles parallèles au cercle de l'équateur, les cercles connus par les cercles méridiens, de sorte que ce qui se produit soit conforme à ce qui apparaît sur la sphère solide." 8

Le but de Ptolémée est clair: trouver un procédé pour représenter certaines lignes de la sphère céleste sur un plan, sans qu'il soit question de l'étude géométrique de ce procédé. Et de fait, Ptolémée conçoit la projection stéréographique d'une sphère sur un plan, mais les problèmes soulevés par le tracé de ces lignes exigent une étude géométrique de la dite projection, étude que Ptolémée semble avoir ignorée. Pappus l'avait-il faite dans son commentaire du Planispbère? On n'en sait rien: le texte de Pappus ne nous est jamais parvenu, non plus que sa traduction arabe. Telle est la situation jusqu'au $\mathrm{IX}^{\mathrm{e}}$ siècle.

$\mathrm{Au} \mathrm{Ix}$ siècle on parvient en effet à une découverte considérable, celle du point de vue des transformations en géométrie. Cette découverte, pour le

7 Acta Eruditorum, 1696, p. 269.

8 Fi tastib basit al-kura, ms. Istanbul, Aya Sofia 2671, fol. 1. 
dire vite, s'est faite presque naturellement, et, en tout cas, indépendamment, lors de l'étude de deux groupes de questions. Il s'agit d'abord de ces questions qui surgirent au cours de la recherche sur les coniques, sur les aires de certains segments elliptiques et paraboliques, et sur la génération de certaines courbes. Le second groupe comporte en revanche des questions rencontrées par les astronomes, et que nous avons déjà évoquées à propos de Ptolémée: celles qui touchent à la représentation exacte de la sphère céleste. Ces questions s'imposent avec d'autant plus de force que l'on s'intéresse activement à la construction des astrolabes. L'astrolabe, on le sait, est un instrument destiné à étudier la sphère céleste, animée d'un mouvement de rotation autour d'un axe, par projection sur une surface mobile superposée à une surface fixe. Il permet donc la représentation exacte de la sphère céleste, et de la position des étoiles en fonction de leur hauteur sur l'horizon. Il servait à déterminer les heures, la longitude et la latitude célestes des astres, la direction de La Mecque, l'ascension des étoiles ... ; autrement dit, il répondait aux besoins de l'astronomie, de la géographie, de l'astrologie, de la religion, de la médecine, etc. On n'insistera jamais assez sur un phénomène bien nouveau, au $\mathrm{IX}^{\mathrm{e}}$ siècle: un progrès sans précédent de la construction des astrolabes et de leur usage. À son tour, la demande sociale d'astrolabes a suscité la multiplication des recherches sur les projections, utiles à leur construction. Ainsi, pour ne prendre qu'un exemple au milieu du $\mathrm{IX}^{\mathrm{e}}$ siècle, le philosophe et savant al-Kindi, les mathématiciens et astronomes, les frères Banu Musa, l'astronome al-Farghani, étudiaient les projections et engageaient la première controverse sur la valeur respective de chacune. Quant à l'astronome alFarghani ${ }^{9}$, il invente une projection zénithale équidistante, rapportée à l'un des pôles de l'écliptique. Cette projection est très proche de celle inventée par Lambert plus tard, et, ensuite, par Cagnoli. C'est encore al-Farghani qui donne le premier exposé théorique de la projection stéréographique. Il démontre en outre sa propriété fondamentale: cette projection transforme les cercles passant par le pôle en droites, et les cercles ne passant par le pôle en cercles.

Il n'est pas question de faire ici l'histoire de ces travaux aux $\mathrm{IX}^{\mathrm{e}}$ et $\mathrm{x}^{\mathrm{e}}$ siècles. Je rappelle simplement que la recherche était intense, multiple et continue; on ne s'y arrêtait plus à la projection stéréographique, mais on inventa d'autres projections. Cette recherche a abouti vers la fin du $\mathrm{x}^{\mathrm{e}}$ siècle à la constitution d'un nouveau chapitre de géométrie: à ce chapitre, on associe les noms

9 Al-Kamil, mssLondres, British Library, Or 5479 , fol. $38^{\mathrm{v}}-40^{\mathrm{v}}$ L'édition et la traduction, ainsi que son commentaire sont à paraître dans R. Rashed, "Al-Farghani: On Geometry". 
des deux mathématiciens, al-Quhi et Ibn Sahl ${ }^{10}$. Je voudrais un peu insister sur ce point.

Al-Quhi a rédigé un livre intitulé L'art de l'astrolabe par la démonstration ${ }^{11}$ Comme le suggère le titre, l'auteur ne s'intéresse pas aux problèmes pratiques qui pouvaient se poser aux artisans constructeurs d'astrolabes, ou aux utilisateurs de l'astrolabe: il ne considère que la théorie géométrique sous-jacente à cette construction. Al-Quhi se livre donc, dès le début de son traité, à l'étude générale de la projection d'une sphère d'axe connu sur une surface, qui peut être, ou non, de révolution. Cette étude, à son tour, l'amène à distinguer deux cas pour la surface de révolution, selon que son axe est, ou non, parallèle à l'axe de la sphère. Aussi al-Quhi a-t-il été amené à définir les projections cylindriques - de direction parallèle ou non à l'axe de la sphère - et les projections coniques à partir d'un sommet appartenant ou n'appartenant pas à cet axe. On ne s'arrête donc plus à la seule projection stéréographique, celle qui est nécessaire à la construction de l'astrolabe, mais on invente et on étudie d'autres projections indépendantes de l'astrolabe: projections coniques, dont le pôle n'est plus situé sur la sphère, projections cylindriques, orthographiques ou non.

Aussi importante que ces nouveaux concepts est leur présentation: ils interviennent comme les éléments d'un exposé sur la méthode des projections. Sans doute suscité par les problèmes posés par la construction de l'astrolabe, ce discours général est aussi élaboré indépendamment d'eux. C'est ainsi qu'al-Quhi prend délibérément le parti - qu'il justifie - de négliger les cas qu'il a pris soin de définir: la projection cylindrique de direction non parallèle à l'axe de la sphère ; et la projection conique de sommet non situé sur la sphère. Autrement dit, alQuhi a introduit les différents types de projection, dont un seul sera utilisé pour l'astrolabe. Pour dégager ce trait de la recherche géométrique, considérons les différentes étapes parcourues, à partir d'al-Quhi, par son contemporain Ibn Sahl.

Ibn Sahl n'étudie pas seulement ces projections ; il examine aussi comment, selon les différents cas, la surface mobile de l'astrolabe peut tourner tout en restant superposée à la surface fixe. Il commence par considérer le cas où la surface de l'astrolabe est un plan: toute perpendiculaire à ce plan est alors un axe pour ce plan. Il envisage deux situations, selon que l'axe de la sphère et l'axe de la surface sont ou non confondus. Considérons à titre d'exemple le cas où les deux axes sont confondus. À la suite d'al-Quhi, mais de manière plus élaborée, Ibn Sahl introduit les concepts suivants:

10 R. Rashed, Géométrie et Dioptrique au X $X^{\ell}$ siècle: Ibn Sabl, al-Qubi et Ibn al-Haytham, Paris, 1993.

11 Ibid., Appendice III. 
1) Projection cylindrique de direction donnée, parallèle à l'axe ;

2) projection cylindrique de direction donnée, non parallèle à l'axe ;

3) projection conique à partir d'un point donné sur l'axe ;

4) projection conique à partir d'un point donné qui n'est pas sur l'axe.

Notons que, dans un autre traité intitulé Sur les propriétés de trois sections. coniques, le même Ibn Sahl étudie la division harmonique. Il s'écarte alors d'Apollonius, et, au lieu de caractériser, comme ce dernier, les divisions harmoniques. par l'égalité de deux rapports, il donne la relation rapportée au milieu de l'un des couples conjugués, pris comme origine. Or la division harmonique se conserve par projection cylindrique ou conique, qui sont les deux projections étudiées par Ibn Sahl.

On voit, sans qu'il faille entrer davantage dans les détails, comment au $\mathrm{x}^{\mathrm{e}}$ siècle, à partir des préoccupations astronomiques, on est parvenu à la découverte du point de vue projectif: l'étude des projections cylindriques et coniques de la sphère, de ses points, de ses diamètres, de ses cercles et des figures qui sont tracées sur elle. Tout comme le commentaire d'Ibn Sahl, le traité d'al-Quhi commence, je l'ai rappelé, par un exposé de ces projections et de leurs propriétés, indépendamment de l'astrolabe, pour ensuite passer aux problèmes résolus par les projections stéréographiques, et qui pouvaient se poser, théoriquement au moins, lors de la construction et de l'utilisation de l'instrument. Ce clivage qui partage l'exposé entre une partie entièrement consacrée aux projections, mais de la sphère seulement, et une seconde partie relative aux problèmes qui pouvaient se poser pour l'astrolabe, marque bien les limites de l'autonomie de ce chapitre par rapport à son terrain d'origine. Autre survivance de ce lieu d'origine, le privilège accordé au problème inverse: au lieu de partir de la sphère projetée, on part au contraire de sa représentation.

Ce nouveau chapitre de la géométrie se distingue également par son langage et par les procédés de démonstration mis en œuvre. Le langage est mixte: au vocabulaire de la théorie des proportions, celui de la géométrie traditionnelle, se mêlent des termes qui désormais désignent les concepts projectifs. Les démonstrations sont elles aussi composées de comparaisons de rapports, tout comme dans la géométrie traditionnelle, mais aussi de rabattements. Lorsque par exemple al-Quhi établit la propriété suivante: à tout cercle tracé sur la sphère, et dont le plan ne contient pas le pôle, la projection stéréographique associe un cercle dans le plan de projection, et inversement; le mathématicien utilise à cet effet la proposition 1. 5 des Coniques d'Apollonius, proposition qui étudie la section d'un cône à base circulaire par un plan, dans le cas où le plan de base et le plan sécant sont des plans antiparallèles. L'idée de l'inversion n'effleure pas en- 
core cet auteur, ni du reste ce fait que la projection stéréographique est la restriction d'une inversion dans l'espace. Mais il reste qu'al-Quhi a fréquemment recours à la technique de rabattement. Il résout le plus souvent le problème à l'aide de la propriété suivante: un point quelconque, son homologue et le pôle de projection sont alignés. Il fait alors appel à plusieurs reprises à des rabattements, qui permettent des constructions en géométrie plane.

Ainsi, qu'il s'agisse de son domaine, de son langage ou des méthodes de démonstration qui s'y déploient, ce chapitre de géométrie conçu par les mathématiciens $\mathrm{du} \mathrm{x}^{\mathrm{e}}$ siècle est issu des problèmes de l'astrolabe, auxquels on avait commencé à répondre plus d'un siècle auparavant. Il s'agit d'un chapitre de géométrie projective, dont les mathématiciens du siècle suivant - comme par exemple al-Biruni - ne cesseront de s'occuper. Mais à la différence des recherches sur les isopérimètres, ce chapitre n'avait jamais rompu les liens qui l'unissent à son terrain d'origine. Cette rupture ne sera consommée qu'au prix d'une accumulation d'autres méthodes projectives, obtenues à l'occasion d'autres recherches: l'étude de la perspective, des coniques, etc.; c'est-à-dire dans les travaux de Kepler, Desargues, Pascal, La Hire, Newton, et bien d'autres encore avant Poncelet.

Il ressort des exemples précédents que les chapitres les plus avancés des mathématiques anciennes et classiques semblent bien être originairement liés à l'activité théorique ou pratique de l'astronomie. Rappelons, pour ne retenir que les deux derniers exemples, que l'extrémalité de la sphère et du cercle était une notion cosmologique avant d'être investie d'un vrai statut mathématique, et que la projection stéréographique était un procédé pour tracer une carte du ciel avant d'être conçue comme transformation géométrique. L'extrémalité de la sphère et du cercle devient un objet véritablement mathématique lorsque l'on commence à l'insérer dans un réseau déjà complexe de définitions, d'axiomes et d'opérations divers, qui lui ôtent tout caractère d'unicité: telle est la première étape. Dans un deuxième temps, le cercle lui-même devient une limite des polygones, et la sphère une limite des polyèdres. Ainsi s'établit une double distance avec les objets d'origine. Il en est de même pour la projection stéréographique: pour qu'elle devienne une vraie transformation géométrique, il faut attendre qu'elle soit conçue comme une projection conique de pôle l'un des points de la sphère, soit sur un plan tangent à la sphère au point diamétralement opposé, soit sur un plan parallèle à celui-ci. Cette transformation n'a plus rien de propre à l'astronomie, même si celle-ci demeure l'un de ses domaines privilégiés d'application. Dans les deux cas évoqués, comme dans les autres, la distance qui sépare doublement de l'origine s'établit à deux niveaux différents: l'objet est reformulé pour transcender l'objet astronomique de départ ; la discipline - isopérimétrie, projections, 
coniques ... - est, en quelque sorte, le fossoyeur de ses origines, pour commencer, ou recommencer.

Notons en conclusion que, au moins pour ces chapitres, l'idéalité mathématique semble bien se fonder sur une autre idéalité, qui permet déjà de parler mathématiquement des organons ou des contours des phénomènes; de sorte que la notion d'origine est déjà trop abstraite et trop dense pour se prêter à une description eidétique, et encore moins à une investigation archéologique, quel que soit le sens que l'on donne à cette formule. 